\title{
Large-scale flows under location uncertainty: a consistent stochastic framework
}

\author{
Chapron Bertrand ${ }^{1}$, Derian P. ${ }^{2}$, Memin E. ${ }^{2,}$, Resseguier Valentin ${ }^{1,2}$ \\ ${ }^{1}$ IFREMER, LOPS, Plouzane, France. \\ ${ }^{2}$ INRIA, IRMAR, Campus Univ Beaulieu, Rennes, France. \\ * Corresponding author : E. Memin, email address : etienne.memin@inria.fr
}

\begin{abstract}
:
Using a classical example, the Lorenz-63 model, an original stochastic framework is applied to represent large-scale geophysical flow dynamics. Rigorously derived from a reformulated material derivative, the proposed framework encompasses several meaningful mechanisms to model geophysical flows. The slightly compressible set-up, as treated in the Boussinesq approximation, yields a stochastic transport equation for the density and other related thermodynamical variables. Coupled to the momentum equation through a forcing term, the resulting stochastic Lorenz-63 model is derived consistently. Based on such a reformulated model, the pertinence of this large-scale stochastic approach is demonstrated over classical eddy-viscosity based large-scale representations.
\end{abstract}

Keywords: large-scale flow modelling, stochastic parametrization, modelling under location uncertainty, stochastic Lorenz model, stochastic transport 


\section{Introduction}

Today, in their most common expression, large-scale geophysical flow representations rely on the Reynolds decomposition and the inclusion of a subgrid dissipative model to represent the action of numerically non-resolved components over the resolved scales. Most used subgrid models heavily bank on the eddy viscosity concept - also called Boussinesq assumption (Boussinesq 1877) - built upon a straight analogy with the molecular dissipation mechanism. The celebrated Smagorinsky model (Smagorinsky 1963) is one of the most representative instance of such models. The eddy viscosity concept is essential to achieve a numerical stability in draining the energy accumulated at the cutoff resolution through the direct energy cascade process. Its pure dissipative behavior further prevent to take into account local backscattered energy or inhomogeneous turbulence.

This article has been accepted for publication and undergone full peer review but has not been through the copyediting, typesetting, pagination and proofreading process, which may lead to differences between this version and the Version of Record. Please cite this article as doi: $10.1002 /$ qj.3198 
To represent the large-scale evolution of turbulent fluid flows, a different strategy can be envisaged, considering the decomposition of the flow into a large-scale smooth component and a fast oscillating velocity component modeled as a random field (seen as decorrelated at large scales). Yet, such a decomposition requires to modify the material derivative through the introduction of a stochastic transport operator (Mémin 2014; Resseguier et al. 2017a).

One advantage of this framework over the Reynolds decomposition lies in its ability to deal with non-smooth expressions of the small-scale component at the resolved time scale. It further introduces, without any supplementary assumption, the following mechanisms: (i) a dissipative operator directly related to the mixing effect of the large-scale components by the small-scale velocity; (ii) a multiplicative noise representing small-scale energy backscattering; and (iii) a modified advection term related to the so-called turbophoresis phenomena, associated to the migration of inertial particles in regions of lower turbulent diffusivity (Reeks 1983). Those properties have already been used to define data-driven inhomogeneous subgrid models to stabilize reduced order flow models in eapturing the principal local dissipation directions and the small-scale induced advection field (Resseguier et al. 2017d). Corresponding dy-viscosity models are not any more constant, but adapted to the dynamics. This random framework also enables to derive stochastic namics from the very same physical conservation principles as in the deterministic case and is amenable to the usual geophysical ling approximations (Resseguier et al. 2017b,c).

In this work, this representation is applied to the famous Lorenz-63 model to illustrate the pertinence of such a consistent stochastic - representation over a classical eddy diffusivity model. In particular, it is shown for the Lorenz-63 that a classical eddy-viscosity modelling strongly slows down the exploration of the attractor, while the stochastic approach provides a much faster exploration.

\section{Stochastic representation of the Lorenz-63 model}

The celebrated Lorenz-63 model (Lorenz 1963) corresponds to the description of an incompressible flow undergoing a Rayleigh-Bénard convection caused by a temperature gradient between the bottom and the top of the fluid domain. It aims at representing atmospheric convection in a $2 \mathrm{D}$ simplified way.

The Lorenz-63 model is formally derived from the Boussinesq approximation - i.e. small density variations - of the Navier-Stokes equations. Its complete derivation is described in Lorenz original paper (Lorenz 1963) or given in greater details in the book Berge

al. (1987). To derive its stochastic representation, we closely follow the same derivation. Yet, we start from a stochastic Boussinesq sistem, derived itself from physical conservation principles and a stochastic representation of the flow. Such representation, termed as modeling under location uncertainty, has been recently proposed in Mémin (2014); Resseguier et al. (2017a), and is hereafter outlined. Note that similar models could be derived from Hamiltonian principles as described in Holm (2015).

Flow modeling under location uncertainty

the modeling under uncertainty the model errors are introduced at the lowest level of the dynamics. The basic idea is built on the ssumption that the Lagrangian fluid particles displacement, $\boldsymbol{X}_{t}$, results from a smooth velocity component, $\mathbf{v}$, and a fast oscillating dom field uncorrelated in time. At time $t$, the location of a fluid particle initially located at $\boldsymbol{X}_{t_{0}}$ is:

$$
\boldsymbol{X}_{t}=\boldsymbol{X}_{t_{0}}+\int_{t_{0}}^{t} \mathbf{v}\left(X_{s}, s\right) \mathrm{d} s+\int_{t 0}^{t} \boldsymbol{\sigma}\left(X_{s}, s\right) \mathrm{d} \boldsymbol{B}_{s}
$$

which reads also in a more compact differential form as:

$$
\mathrm{d} \boldsymbol{X}_{t}=\mathbf{v}\left(\boldsymbol{X}_{t}, t\right) \mathrm{d} t+\boldsymbol{\sigma}\left(\boldsymbol{X}_{t}, t\right) \mathrm{d} \boldsymbol{B}_{t} .
$$


The solenoidal (possibly inhomogeneous) random field, representing the small-scale velocity component, is build through the application of a linear operator, $\boldsymbol{\sigma}$, to a space-time white noise, $\mathrm{d} \boldsymbol{B}_{t}$. It is explicitly defined on a spatial domain $\Omega$ from a kernel, $\breve{\boldsymbol{\sigma}}$, as:

$$
\boldsymbol{\sigma}(\boldsymbol{x}, t) \mathrm{d} \boldsymbol{B}_{t}=\int_{\Omega} \breve{\boldsymbol{\sigma}}(\boldsymbol{x}, \boldsymbol{y}, t) \mathrm{d} \boldsymbol{B}_{t}(\boldsymbol{y}) \mathrm{d} \boldsymbol{y}
$$

The kernel, $\breve{\boldsymbol{\sigma}}$, (or the operator $\boldsymbol{\sigma}$ ) encodes the random field spatial correlations, whereas the white noise function specifies its temporally decorrelated character.

Decomposition (2) leads to a stochastic representation of both the Reynolds transport theorem (RTT) and the material derivative, $D_{t}$ (derivative along the flow). When the material derivative of a quantity is deterministic, (such as in the case of a conservation constraint, for instance) this derivative coincides with the stochastic transport operator, $\mathbb{D}_{t}$, defined for any field $\Theta$ as:

$$
\mathbb{D}_{t} \Theta=\mathrm{d}_{t} \Theta+\left(\mathbf{v}^{\star} \mathrm{d} t+\boldsymbol{\sigma} \mathrm{d} \boldsymbol{B}_{t}\right) \cdot \nabla \Theta-\nabla \cdot\left(\frac{1}{2} \boldsymbol{a} \nabla \Theta\right) \mathrm{d} t
$$

involving the time increment term $\mathrm{d}_{t} \Theta=\Theta(\boldsymbol{x}, t+\mathrm{d} t)-\Theta(\boldsymbol{x}, t)$, as $\Theta$ is a non differentiable random function. This function depends hong other things on the particles driven by the Brownian component flowing through a given point. The diffusion matrix, $\boldsymbol{a}$, is solely

- aned by the one-point one-time covariance of the unresolved displacement per unit of time:

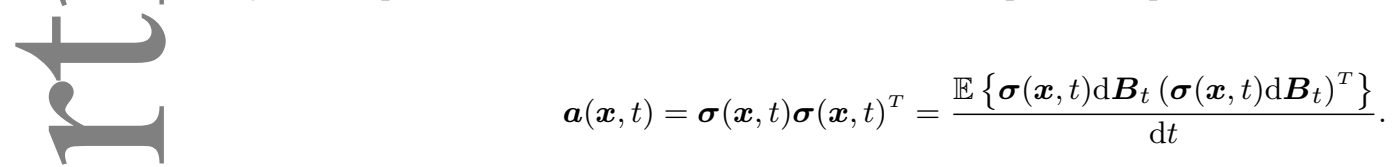

This quantity corresponds to the diagonal of the covariance tensor and has the dimension of an eddy viscosity term (with units in $\left.\mathrm{s}^{-1}\right)$. The modified drift is given by

$$
\mathbf{v}^{\star}=\mathbf{v}-\frac{1}{2}(\boldsymbol{\nabla} \cdot \boldsymbol{a})^{T}
$$

A derived, both the stochastic RTT and material derivative involve a diffusive subgrid term, a multiplicative noise and a modified advection drift induced by the small-scale inhomogeneity. This material derivative has the remarkable property to conserve the energy of any randomly transported tracer realization (Resseguier et al. 2017a):

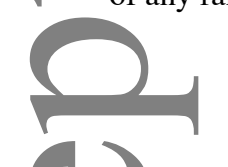

$$
\begin{aligned}
& \text { (1) } \\
& \frac{\mathrm{d}}{\mathrm{d} t} \int_{\Omega} \Theta^{2}=\int_{\Omega} \mathbb{D}_{t} \Theta^{2}=0
\end{aligned}
$$

Given the RTT, the classical conservation laws of mechanics (linear momentum, energy, mass) can be expressed within a stochastic flow form (2). It should be noted that an incompressible homogeneous noise, i.e. with a divergence-free diffusion tensor $\boldsymbol{\nabla} \cdot \boldsymbol{\sigma}(\boldsymbol{x}, t)=0$, d fined over a periodic domain for simplicity, leads to a constant diffusion matrix. In that case, the effective advection reduces to the rarge-scale drift component, and the diffusive subgrid term boils down to weighted second order partial derivatives.

The modeling under location uncertainty thus conveys a practical alternative to the design of stochastic representations geophysical flows. Various techniques have already been considered such as homogeneization, stochastic modes reduction, renormalization closure, a posteriori random forcing, or parameter random perturbation (see Berner et al. (2017); Franzke et al. (2015) and references therein for a review). However, the modeling under location uncertainty unambiguously provides a rigorous physical derivation of the stochastic system that directly stems from the conservation principles. It then facilitates the set-up of classical 
scaling procedures to include all the ingredients needed for a large scale representation, e.g. subgrid diffusion, modified advection akin to turbophoresis phenomenon and backscattering. To obtain similar stochastic Eulerian equations, Holm (2015) relies on geometric mechanics and a variational principle to propose an alternative construction. This latter formulation is helicity preserving, whereas the modeling under uncertainty conserves the energy. Those stochastic models have been recently justified through the homogeneization of multiscale Lagrangian dynamics Cotter et al. (2017), and when restricted to a 3D (energy conserving) Euler model, Crisan et al. (2017) demonstrates that analytical properties of the 3D deterministic Euler equations are also preserved by such stochastic representations.

\subsection{Boussinesq system under location uncertainty}

Following the location uncertainty principle, stochastic Navier-Stokes and Boussinesq models have been derived by Mémin (2014) and

R sseguier et al. (2017a), respectively. In a 2D inertial frame of reference indexed by the horizontal and vertical coordinates $x$ and $z$, an incompressible anisotropic homogeneous random field (i.e. with $\boldsymbol{\nabla} \cdot \breve{\boldsymbol{\sigma}}\left(\boldsymbol{x}-\boldsymbol{x}^{\prime}\right)=0$ ) is characterized by a constant diagonal diffusion tensor $\boldsymbol{a}=\operatorname{diag}\left(\mathrm{a}_{\mathrm{x}}, \mathrm{a}_{\mathrm{z}}\right)$. Accordingly, the momentum equation of the large scale velocity $\mathbf{v}=(u, w)^{T}$ reads:

$$
\rho\left(\partial_{t} \mathbf{v}+(\mathbf{v} \cdot \boldsymbol{\nabla}) \mathbf{v}-\frac{1}{2} \sum_{i} a_{i} \partial_{i i}^{2} \mathbf{v}\right)=\rho \boldsymbol{g}-\boldsymbol{\nabla} P+\mu \boldsymbol{\nabla}^{2} \mathbf{v}
$$

where with usual notations $\mu$ denotes the dynamic viscosity, $P$ is the pressure, and $\boldsymbol{g}$ the gravity force. Thanks to the homogeneous structure of the noise component, these equations closely resemble a large-scale model with proper constant eddy viscosity coefficients along the horizontal and vertical directions. Yet, this system is complemented by a stochastic thermo-dynamical equation describing the temperature evolution:

$$
\mathbb{D}_{t} T=D_{T} \Delta T \mathrm{~d} t
$$

A previously discussed (7), when the thermal diffusion coefficient $D_{T}$ is negligible, the temperature is transported and its norm is preserved. In the steady non-convective state (when the fluid is at rest), the temperature varies linearly with the domain depth $h$ : $x, z, t)=T_{b}-\frac{z}{h} \delta T$, where $\delta T=T_{b}-T_{u}$ is positive as the bottom temperature $T_{b}$ is higher than the temperature at the top $T_{u}$. The de viation from this linear model is:

$$
\tau(x, z, t)=T(x, z, t)-T_{b}+\frac{z}{h} \delta T
$$

and Its evolution obeys the following stochastic evolution law:

$$
\mathbb{D}_{t} \tau-w \frac{\delta T}{h} \mathrm{~d} t-\frac{\delta T}{h}\left(\boldsymbol{\sigma} \mathrm{d} \boldsymbol{B}_{t}\right)_{z}=D_{T} \nabla^{2} \tau \mathrm{d} t
$$

This latter model introduces a random transport of the temperature fluctuation, together with deterministic and random forcing of the rtical velocity component. Writing the density variations in power of temperature fluctuation $\left(T-T_{b}\right)$, to leading order we may ite $\rho(T)=\rho_{0}-\alpha \rho_{0}\left[-\frac{z}{h} \delta T+\tau\right]$, where $\rho_{0}=\rho\left(T_{b}\right)$ and the thermal expansion coefficient is $\alpha=-\frac{1}{\rho_{0}} \frac{\partial \rho}{\partial T}$. Under the assumption of negligible compressibility, the Boussinesq approximation states that the density variations can be ignored in the momentum equation (8) and only kept within the gravity term. This system further simplifies through the hydrostatic equilibrium, which holds for the fluid at rest:

$$
\partial_{t} \mathbf{v}+(\mathbf{v} \cdot \boldsymbol{\nabla}) \mathbf{v}-\frac{1}{2} \sum_{i} a_{i} \partial_{i i}^{2} \mathbf{v}=-\alpha \tau \boldsymbol{g}-\frac{1}{\rho_{0}} \nabla P+\nu \nabla^{2} \mathbf{v}
$$


where $\nu$ denotes the kinematic viscosity. First, the formulation is adimensionalized with respect to the time variable $t^{\prime}=\frac{D_{T}}{h^{2}} t$, where $h^{2} / D_{T}$ corresponds to the typical time of a thermal diffusion over $h$, and the spatial variables: $x^{\prime}=x / h, z^{\prime}=z / h$. The uncertainty ratio $\Upsilon$, characterizing the order of magnitude of the horizontal turbulent diffusion $a_{x}^{\prime}=\Upsilon a_{x}, a_{z}^{\prime}=\Upsilon a_{z}$, is then introduced, together with the temperature deviation $\tau^{\prime}=\tau / \delta t$. Multiplying the system by $\frac{h^{3}}{D_{T} \nu}$ and finally incorporating the dimensionless Prandtl number $P_{r a}=\frac{\nu}{D_{T}}$, ratio of the kinematic viscosity to the thermal diffusivity, the Rayleigh number $R a=\frac{\alpha \delta T g h^{3}}{\nu D_{T}}$ and the dimensionless pressure variable $\Pi=\frac{1}{\rho_{0}} \frac{h^{2}}{D_{T} \nu} \partial_{z^{\prime}} P^{\prime}$, the following $2 \mathrm{D}$ system is obtained (where the prime index has been dropped for sake of clarity):

$$
\begin{aligned}
& \frac{1}{P r a}\left(\partial_{t} \mathbf{v}+(\mathbf{v} \cdot \nabla) \mathbf{v}-\frac{1}{2 \Upsilon} \sum_{i} a_{i} \partial_{i i}^{2} \mathbf{v}\right)=-\nabla \Pi+\nu \nabla^{2} \mathbf{v} \\
& \mathbb{D}_{t} \tau-w \mathrm{~d} t-\Upsilon^{-1 / 2}\left(\boldsymbol{\sigma} \mathrm{d} \boldsymbol{B}_{t}\right)_{z}=\nabla^{2} \tau \mathrm{d} t
\end{aligned}
$$

10. $\mathrm{S}$ ce $\mathbf{v}(x, z)=(u(x, z), w(x, z))$ is divergence-free, the $2 \mathrm{D}$ momentum equations can be written in a vorticity stream function form:

$$
\frac{1}{\operatorname{Pra}}\left(\partial_{t} \nabla^{2} \Psi+J\left(\Psi, \nabla^{2} \Psi\right)-\frac{1}{2} \frac{1}{\Upsilon} \sum_{i} a_{i} \partial_{i i}^{2} \nabla^{2} \Psi\right)=R a \partial_{x} \tau+\nu \nabla^{4} \Psi
$$

- where $\Psi$ denotes the stream function and $J(\Psi, \omega)=\nabla^{\perp} \Psi \cdot \nabla \omega=\mathbf{v} \cdot \nabla \omega$ denotes the Jacobian of the transformation $\boldsymbol{x} \rightarrow(\Psi, \omega)^{T}$ 104 w the $2 \mathrm{D}$ vorticity $\omega=\nabla^{\perp} \cdot \mathbf{v}=-\partial_{z} u+\partial_{x} w$. This equation together with the thermal equation describes the whole dynamics of 05 the flow. As for the divergence-free random field, we similarly consider a stream function vector formulation $\boldsymbol{\sigma}^{\mathrm{d}} \boldsymbol{B}_{t}=\boldsymbol{\nabla}^{\perp} \boldsymbol{\Psi}_{\sigma}^{T} \mathrm{~d} \boldsymbol{B}_{t}$. 106 Note that the kernel, $\breve{\Psi}_{\sigma}$, of the linear operator $\Psi_{\sigma}$ is a vector of two components:

$$
\left(\boldsymbol{\Psi}_{\sigma}^{T} \mathrm{~d} \boldsymbol{B}_{t}\right)(\boldsymbol{x})=\int_{\Omega} \mathrm{d} \boldsymbol{z} \sum_{k=1}^{2}\left(\breve{\Psi}_{\sigma}(\boldsymbol{x}, \boldsymbol{z})\right)_{k}\left(\mathrm{~d} \boldsymbol{B}_{t}(\boldsymbol{z})\right)_{k} .
$$

\section{Fourier modes projection and simplified solution}

108 The Lorenz model corresponds to a simplified solution of this system, considering a Galerkin projection onto the first Fourier modes 10. $c$ apled with suited boundary conditions. The boundary conditions are $\tau=0$ at $z=0,1$ to get the appropriate fixed temperature on the $110 \mathrm{~d} d$ main frontiers and $\partial_{z} u=0$ and $w=0$ at $z=0,1$, i.e. neglecting the shear forces on the boundary. For the random term, we assume neriodic boundary conditions for simplicity. Indeed, it is a necessary condition for homogeneity, and thus for a constant diagonal no diucilon tensor. These specific boundary conditions may be understood as a random forcing at the domain boundary, and are satisfied Dy the following ansatz for the streamfunction and the temperature deviation:

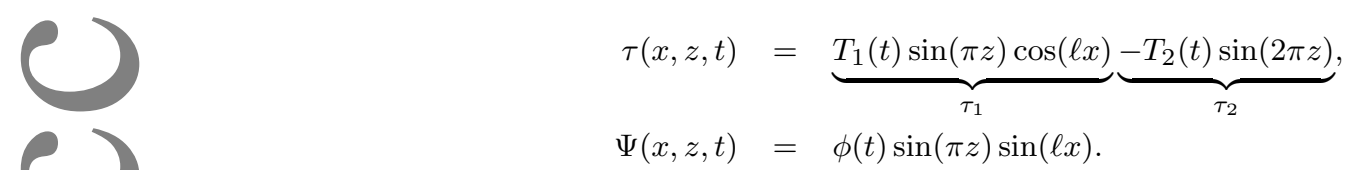

$\Psi(x, z, t)=\phi(t) \sin (\pi z) \sin (\ell x)$.

e two parts, $\tau_{1}$ and $\tau_{2}$ of the temperature fluctuation are random (through $T_{1}$ and $T_{2}$ ) and provide the temperature deviation on the fluid parcel boundary and at the parcel center, respectively. To ensure a diagonal diffusion tensor as previously specified, the random 116 stream function term is defined on the two first Fourier modes and their conjugates :

$$
\boldsymbol{\Psi}_{\sigma}^{T} \mathrm{~d} \boldsymbol{B}_{t}=\frac{1}{\Upsilon^{1 / 2}} \sum_{i, j=1}^{2}\left(\cos \left(g_{i j}\right) \mathrm{d} \beta_{t}^{i j}+\sin \left(g_{i j}\right) \mathrm{d} \zeta_{t}^{i j}\right),
$$


where the phases are given as $g_{i j}=(-1)^{i} \pi z-(-1)^{j} \ell x$ and the modulus are defined from the constant $\Upsilon^{-1 / 2}$ and eight independent scalar Brownian variables $\left\{\beta_{t}^{i j}, \zeta^{i j}: i, j=1,2\right\}$. It may be checked that this representation fits the homogeneity condition:

$$
\mathbb{E}\left\{\boldsymbol{\Psi}_{\sigma}^{T}\left(x_{1}, z_{1}\right) \mathrm{d} \boldsymbol{B}_{t} \boldsymbol{\Psi}_{\sigma}^{T}\left(x_{2}, z_{2}\right) \mathrm{d} \boldsymbol{B}_{t}\right\}=\frac{1}{\Upsilon} \sum_{i, j=1}^{2} \cos \left((-1)^{i} \pi\left(z_{1}-z_{2}\right)-(-1)^{j} \ell\left(x_{1}-x_{2}\right)\right) \mathrm{d} t .
$$

The small-scale random velocity becomes:

$$
\begin{aligned}
\Upsilon^{1 / 2} \boldsymbol{\sigma} \mathrm{d} \boldsymbol{B}_{t} & =\nabla^{\perp} \boldsymbol{\Psi}_{\sigma}(x, z) \mathrm{d} \boldsymbol{B}_{t} \\
& =\sum_{i, j=1}^{2}\left(\begin{array}{c}
(-1)^{i} \pi \\
(-1)^{j} \ell
\end{array}\right)\left(\sin \left(g_{i j}\right) \beta_{t}^{i j}-\cos \left(g_{i j}\right) \zeta_{t}^{i j}\right),
\end{aligned}
$$

ich can be developed in factors of $\cos x \sin , \cos x \cos$, and $\sin x \sin$. We may check that the diffusion tensor is then given by

$$
\boldsymbol{a}=\frac{1}{\Upsilon} \sum_{i, j=1}^{2}\left(\begin{array}{cc}
(-1)^{2 i} \pi^{2} & (-1)^{i+j} \pi \ell \\
(-1)^{j+i} \pi \ell & (-1)^{2 j} \ell^{2}
\end{array}\right)=\frac{4}{\Upsilon}\left(\begin{array}{cc}
\pi^{2} & 0 \\
0 & \ell^{2}
\end{array}\right)
$$

This choice, though simple, remains sufficiently general for our purpose. Factorizing by $\sin (\pi z) \sin (\ell x)$ the dynamics (14), we (2) mmediately infer that the stream function temporal mode, $\phi(t)$, has to satisfy the following differential equation

$$
\partial_{t} \phi(t)=\frac{\operatorname{PraRaT}(t)}{\left(\ell^{2}+\pi^{2}\right)}-\left(\pi^{2}+\ell^{2}\right)\left(\operatorname{Pra}+\frac{4}{\Upsilon} \frac{\pi^{2} \ell^{2}}{\left(\pi^{2}+\ell^{2}\right)}\right) \phi(t) .
$$

This equation includes a random forcing term coming from the temperature variation. As for the thermo-dynamical equation, removing the high-order frequency terms, gathering on the one hand the terms in $\sin (\pi z) \cos (\ell x)$ and in the other hand the terms in $\sin (2 \pi z)$, and in roducing a new scalar Brownian motion $B_{t}=\frac{1}{2} \sum_{i, j=1}^{2}(-1)^{i+j} \beta_{t}^{i j}$ (for which it can be easily verified that the quadratic variation is $t$ ), we get:

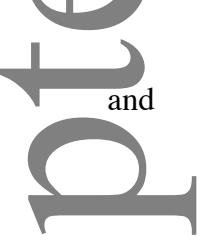

$$
\begin{gathered}
\mathrm{d} T_{1}+\left(\ell^{2}+\pi^{2}+\frac{4}{\Upsilon} \pi^{2} \ell^{2}\right) T_{1}(t) \mathrm{d} t-\ell \phi \mathrm{d} t-\frac{\ell}{\Upsilon^{1 / 2}} \mathrm{~d} B_{t}=-\ell \phi \pi T_{2} \mathrm{~d} t-\frac{\ell \pi T_{2}}{\Upsilon^{1 / 2}} \mathrm{~d} B_{t}, \\
-\mathrm{d} T_{2}-\left(1+\frac{2}{\Upsilon} \ell^{2}\right) 4 \pi^{2} T_{2} \mathrm{~d} t=-\frac{1}{2} \ell \pi \phi T_{1} \mathrm{~d} t-\frac{1}{2} \frac{\ell \pi T_{1}}{\Upsilon^{1 / 2}} \mathrm{~d} B_{t},
\end{gathered}
$$

Lorenz system under uncertainty

get to the final simplified system, we finally consider the time scale $t^{\prime}=\left(\pi^{2}+\ell^{2}\right) t$, the change of variables:

$$
\begin{aligned}
X(t) & =\frac{\ell \pi}{\left(\ell^{2}+\pi^{2}\right) \sqrt{2}} \phi(t), \\
Y(t) & =\frac{r \pi}{\sqrt{2}} T_{1}(t), \\
Z(t) & =\pi r T_{2}(t),
\end{aligned}
$$


and the reduced Rayleigh number

$$
r=\frac{R a \ell^{2}}{\left(\pi^{2}+\ell^{2}\right)^{3}}
$$

With this time renormalized, the new Brownian variable is $B_{t^{\prime}}=\sqrt{\pi^{2}+\ell^{2}} B_{t}$. We also note

$$
\frac{1}{\tilde{\Upsilon}^{1 / 2}}=\frac{2}{\Upsilon^{1 / 2}} \frac{\ell \pi}{\sqrt{\ell^{2}+\pi^{2}} \sqrt{2}} .
$$

Those changes of variables, with the parameter $b=4 \pi^{2} /\left(\pi^{2}+\ell^{2}\right)$, lead to the final system of equations where, for the sake of readability, we kept the notation $\Upsilon$ instead of $\tilde{\Upsilon}$ and dropped the prime index on time:

$$
\begin{aligned}
\frac{\mathrm{d} X}{\mathrm{~d} t} & =\operatorname{Pr}(Y-X)-\frac{4}{2 \Upsilon} X, \\
\mathrm{~d} Y & =\left((r-Z) X-Y-\frac{4}{2 \Upsilon} Y\right) \mathrm{d} t+\frac{r-Z}{\Upsilon^{1 / 2}} \mathrm{~d} B_{t}, \\
\mathrm{~d} Z & =\left(X Y-b Z-\frac{8}{2 \Upsilon} Z\right) \mathrm{d} t+\frac{Y}{\Upsilon^{1 / 2}} \mathrm{~d} B_{t} .
\end{aligned}
$$

-

This model constitutes a stochastic version of the Lorenz model. It is composed of a deterministic differential equation on the velocity variable together with two coupled stochastic differential equations associated with the temperature fluctuations. For a negligible noise $(\Upsilon \rightarrow \infty)$, we recover the original model. Besides, in the $Y$ and $Z$ equations, the noise terms involve the same factors as the advection r $\mathrm{ms}$ in factor of the velocity variable $(X)$. Hence, they both correspond to the advection of temperature variables by the small-scale locity. An additive noise component, weighted by the Rayleigh coefficient, is obtained in the $Y$ equation. It corresponds to the random interaction between the small-scale velocity and the stratification $\delta T$ appearing in (11). This term and its influence on the buoyancy variations has been detailed in Resseguier et al. (2017a).

It should be noticed that in this stochastic Lorenz system, the velocity variable is driven by an ordinary differential equation. This is the first place due to our assumption of a smooth in time large-scale velocity. Relaxing this assumption (i.e. in considering that the ge-scale velocity component depends also on a Brownian variable, which is fully authorized by our derivation) the expression of the locity for the Lorenz 63 system would however remains deterministic. As a matter of fact, the multiplicative noise is antisymmetric, and thus described (in an orthonormal basis) by an antisymmetric matrix with a null diagonal. Consequently, the noise would have no effect, as only one Fourier mode is kept in the Lorenz-63 model to represent the velocity. In other words, the noise transfers energy from one mode to the other. If only one mode is considered, the noise has no effect. In the other hand, the turbulent diffusion is still sent as it takes out energy from the resolved mode to transfer it to the truncated modes.

In the three equations and as compared to the original Lorenz system, the diffusion terms are increased by a factor that depends on the noise variance scale $\Upsilon$. Due to the scale truncation, the energy loss of $Y$ by turbulent diffusion is 4 times larger than the multiplicative ise intake. It is 8 times larger for $Z$. The stochastic system exhibits a symmetry for $(-X,-Y, Z,-B)$. Thus, the law of the solution symmetric for $(-X,-Y, Z)$.

To infer the different physical mechanisms, it is usefull to rewrite system (30) with Stratonovich notations (Oksendal 1998):

$$
\mathrm{d}\left(\begin{array}{l}
Y \\
Z
\end{array}\right)=\boldsymbol{F} \mathrm{d} t+\Upsilon^{-1 / 2} \boldsymbol{G} \mathrm{d} B_{t}=\boldsymbol{F}^{*} \mathrm{~d} t+\Upsilon^{-1 / 2} \boldsymbol{G} \circ \mathrm{d} B_{t}
$$




$$
\begin{aligned}
& \text { where } \boldsymbol{F}^{*}=\boldsymbol{F}-\frac{1}{2 \Upsilon}\left(\boldsymbol{G} \cdot \nabla_{Y Z}\right) \boldsymbol{G} \text {, } \\
& =\underbrace{\boldsymbol{G X}}_{\substack{\text { Large-scale } \\
\text { advection }}}-\underbrace{\left(\begin{array}{cc}
1 & 0 \\
0 & b
\end{array}\right)\left(\begin{array}{c}
Y \\
Z
\end{array}\right)}_{\begin{array}{c}
\text { Molecular } \\
\text { diffusion }
\end{array}}-\underbrace{\left.\frac{1}{2 \Upsilon(} \begin{array}{ll}
3 & 0 \\
0 & 7
\end{array}\right)\left(\begin{array}{l}
Y \\
Z
\end{array}\right)}_{\begin{array}{c}
\text { Turbulent diffusion due } \\
\text { to scale truncation }
\end{array}}, \\
& \boldsymbol{G}=\left(\begin{array}{l}
r \\
0
\end{array}\right)+\left(\begin{array}{cc}
0 & -1 \\
1 & 0
\end{array}\right)\left(\begin{array}{l}
Y \\
Z
\end{array}\right) \text {, and } \nabla_{Y Z}=\left(\begin{array}{c}
\partial_{Y} \\
\partial_{Z}
\end{array}\right) \text {. }
\end{aligned}
$$

Since $\boldsymbol{G}$ represents an advection term, its linear part is antisymmetric and thus has no effect either on the temperature energy $Y^{2}+Z^{2}$ or on the dilation or contraction of the state space $\left(\nabla_{Y Z} \cdot \boldsymbol{G}=0\right)$. However, according to the system's flow Jacobian (Resseguier et al. 2017a), the drift term, $\boldsymbol{F}^{*}$, uniformly shrinks the state space volume:

$$
\begin{aligned}
V(t) & =V(0) \exp \left(\int_{0}^{t}\left(\left(\partial_{X} \dot{X}+\nabla_{Y Z} \cdot F^{*}\right) \mathrm{d} t+\nabla_{Y Z} \cdot \boldsymbol{G} \mathrm{d} B_{t}\right)\right), \\
& =V(0) \exp \left(-\left(P_{r a}+1+b+\frac{7}{\Upsilon}\right) t\right) .
\end{aligned}
$$

150 Note that the noise term increases the shrinking rate through the turbulent diffusion term induced by the spatial scale truncation. In 13 IDI addition, the random terms are volume-preserving since they have an antisymmetric multiplicative structure as stated by the transport erator (4). More arbitrary choices of multiplicative random Lorenz systems studied in the literature do not necessarily keep such 153 pperties Chekroun et al. (2011). This key difference between stochastic systems build from a stochastic transport operator and the multiplicative stochastic system studied in Chekroun et al. (2011) has also been put forward through the Lyapunov exponents in Geurts 155 en al. (2017). While obtained from a different derivation, the stochastic system with noise transport studied in Geurts et al. (2017) is 156 close to the system derived from the modeling under location uncertainty.

157 Additive noise terms in the sytems (Dorfle and Graham 1983) have also been considered. However, those latter models do not 150 correspond to the observed small-scale tracers, which are non-Gaussian and intermittent. Such phenomena are well described in simple 45. Alar advection models with multiplicative random processes (Kraichnan 1968, 1994; Majda and Kramer 1999; Sura et al. 2005). The system expectation (conditionally to the velocity) corresponds to a Lorenz model with an augmented diffusion and hence of Constitutes a damped version of the deterministic version of the original model. There are still three equilibrium points for $r>1$ foc rocan 1 at $(0,0,0)\left( \pm\left[\left(1+\frac{2}{\Upsilon}\right)\left(b+\frac{4}{\Upsilon}\right) \alpha\right]^{1 / 2}, \pm\left[\left(b+\frac{4}{\Upsilon}\right) \alpha\right]^{1 / 2}, \alpha\right)$ with $\alpha=\left(r-\left(1+\frac{2}{\Upsilon}\right)\left(1+\frac{2}{\Upsilon \text { Pra }}\right)\right)$. For a small noise variance, we 16. $\mathrm{r} \epsilon$ over to leading order the usual equilibrium points, but for strong noise, there is a shift due to the large-scale diffusion engendered 164 by the noise.

The classical Lorenz system corresponds to a description of the flow in which the small-scale velocity fluctuations are simply ignored 106 th ough a truncation on the Fourier space. The diffusions introduced are then only related to the kinematic viscosity and to the thermal $16 /$ diffusivity. The modeling of the small-scale effects as purely dissipative processes, as this is done in Large Eddies Simulation (LES), would introduce stronger diffusions through eddy viscosity and eddy diffusivity coefficients.

The Lorenz system under location uncertainty can thus be interpreted as a coarse time-scale description of the dynamical system 170 in which the intrinsic Lagrangian velocity anomaly is encoded through a temporally uncorrelated random variable. Here, the latter 171 is encoded as a scalar white noise variable depicting an uncertainty on the temporal evolution of the two first Fourier modes of the 172 flow velocity. The velocity anomalies have a characteristic time that is much smaller than the resolved (differentiable) velocity. At the 
resolution characteristic time, these velocities anomalies can thus be considered as fully decorrelated. One crucial property of such large scale representations concerns the rate at which they tend to the "finest" original system when the noise tends to zero (e.g. when $\Upsilon \rightarrow \infty)$. We will show in the following section that the proposed stochastic system includes the property to approach the original system for moderates values of $\Upsilon$ and to provide reasonable coarse descriptions for small $\Upsilon$ values. This ability constitute the most striking difference with a diffusive "eddy-viscosity" model, which has a good convergence behavior but yields a wrong representation at high eddy-viscosity value, or with an ad hoc stochastic multiplicative forcing approach that appears to have a poor representation property even for low noise.

\section{Numerical simulations of large-scale representations}

he following, we consider several simulations of this stochastic Lorenz system. The gold standard to which this system should be compared with, would ideally consist to reconstruct an ensemble of trajectories of an equivalent reduced order model built from a full direct numerical simulation of a Raleigh-Benard convection (with a large number of different initial conditions). This solution would istitute a huge computational effort. Instead of doing that, we will compare the performances of different representations of the Lorenz-63 system with the original system. The deterministic Lorenz system does not constitute per se a gold standard in the sense that it corresponds to a reduced order model that represents the evolution of only the first Fourier modes (one mode for the velocity, two modes for the temperature) with no model for the truncated modes. However these modes capture well - in an ideal setting - the recurrent pattern of the metastable Raleigh-Benard convection cells. A representation of the small-scale effects will not considerably atiect the representation of these large scale effects at least in average. Obviously, intermittency and small-scale perturbations will likely modify an instantaneous picture of these cells and of their motion. As a consequence, any Lorenz systems with a representation of the truncated modes should statistically not differ too much from the original Lorenz system. All of them should statistically represent the crme large-scale physics. In particular the pdf or the mean spectrum of the system variables at large scale should be close to those the deterministic Lorenz-63 system. Furthermore beyond its relation with the Raleigh-Benard convection, the Lorenz-63 model is a toy model that reproduces qualitatively essential mechanisms of geophysical dynamics: a temperature advection and a non linearity ith a velocity forced by temperature. It is interesting to observe how different modifications introducing multiplicative noise and dy-diffusion mechanisms depart from the original deterministic system.

We consider the original Lorenz system for the usual chaotic parameters $\left(P_{r} a=10, r=28, b=8 / 3\right.$ ). Its small dimension enables to easily visualize the solution attractor and to obtain empirical probability density function of the phase space. The Lorenz-63 system y 11 be termed with the LZ acronym. For the parameter values investigated here, LZ admits an invariant set, over which almost all initial conditions are attracted.

The second system we will consider corresponds to the dissipative system without the noise terms. This latter system can be in erpreted as a damped version of LZ. The temporal modes are further damped from a supplementary diffusion term, akin to classical large eddies representations of the dynamics through eddy viscosity subgrid models. Expressed as a spatial diffusion in the physical domain, these subgrid effects are represented by a damping term on the Fourier temporal modes. The action of the unresolved variables on the resolved variables solely results from the dissipative subgrid operator. This system will be denoted LES-LZ.

The third model corresponds to the proposed stochastic model. It includes the previous dissipative terms, but also the multiplicative noise terms borne by the location uncertainty formulation. This system is referred to LUS-LZ - for Location Uncertainty Stochastic Lorenz-63. 
(a)

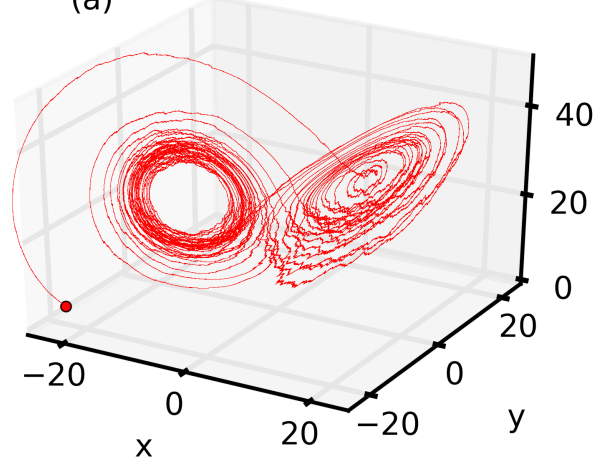

(b)

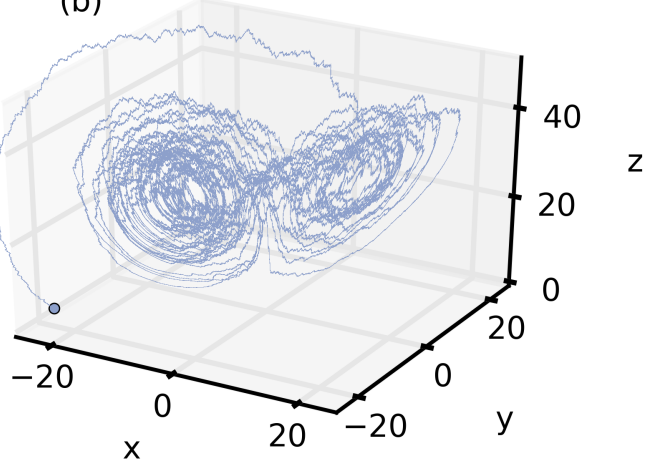

Figure 1. Trajectory of the Lorenz system under location uncertainty (a) and the Basic stochastic Lorenz systems (b) in a strong noise case $(\Upsilon=10)$.

A fourth system is empirically defined by adding to LZ, multiplicative noise variables $1 / \Upsilon Z d B_{t}$ and $1 / \Upsilon Y d B_{t}$ on $Z$ and $Y$ variables,

pectively. Hence the noise has a diagonal structure and there is here no additional diffusion. This basic stochastic model is termed BS-LZ.

A first remark on the different systems can immediately be done. The stochastic and diffusive systems straightforwardly tend to LZ Aen the noise (or the diffusion) tend to zero. Yet, the rate at which those modified systems tend to the deterministic system is crucial. particular, for very small noise condition, it is not desirable to greatly differ from LZ.

For those four systems, simulations with different initial conditions have been carried out. For the two deterministic systems, LZ and the diffusive LES-LZ, an ensemble is engendered by random perturbations of the initial condition. The same point is used to in tiate the realizations of the stochastic systems. The noise amplitude and the initial perturbation have been fixed through the scaling $\Upsilon$. Numerically, the four systems have been set on equal footing. We employed a simple Euler-Maruyama integration for the stochastic di ferential equations associated with a tiny time step $\left(10^{-5}\right)$. To obtain comparable results, an Euler scheme has been used with the same value for the deterministic systems. Several simulations with 100 particles have been run with different noise levels and initial - Anditions. An example of the trajectories of one realization of the two stochastic systems with the same initial condition and the same vel of noise are displayed on Figure 1. The BS-LZ trajectory is rough while the smoother LUS-LZ trajectory is more akin to that of deterministic LZ.

The curves plotted in Figure (2,3 and 4) show, for two different noise levels, the empirical (marginal) probability distribution and the power spectrum of the variables $X, Y$ and $Z$, respectively. As immediately noticed, the diffusive Lorenz system for a strong noise $(\Upsilon=10)$ strongly modifies the empirical pdf. Two peaks are now observed at the equilibrium points, located at the center of the attractor wings. The trajectories are more easily trapped in the attraction bassin of these points. Yet, the eddy viscosity coefficient is smaller an one, $1 / 5$ and $2 / 5$ on $X$ and $Z$, respectively. The spectrum for long time-scale is also modified for the three variables, especially the $Z$ variable. For small diffusion, $\Upsilon=100$, and eddy viscosity coefficients of $1 / 50,1 / 50$ and $1 / 25$ for $X Y$ and $Z$, respectively, the pdfs and the power spectra superimpose almost perfectly with pdfs and spectra of the deterministic system. This model converges to th deterministic system for small diffusion. Note, we recover here the common practice in computer fluid dynamics that limits the use of diffusive LES to resolutions quite close to high resolution simulations. At variance, the random empirically forced system (BS-LZ) performs quite badly. Even for small noise, it leads to significant changes for the pdf shapes of the three variables. Strong discrepancies ah be observed in the spectrum of the $Z$ variable in the transition regions between frequency peaks (fig. 4). The BS-LZ thus badly converge toward the deterministic system. It constitutes a bad random representation of the original system. Compared to the others, the LUS-LZ still holds well for high noise. Though slightly smoothed, the shapes of the marginal pdf and of the spectra are well preserved at large time scale. Some discrepancies only appear at high frequency where the noise impact is clearly visible. 


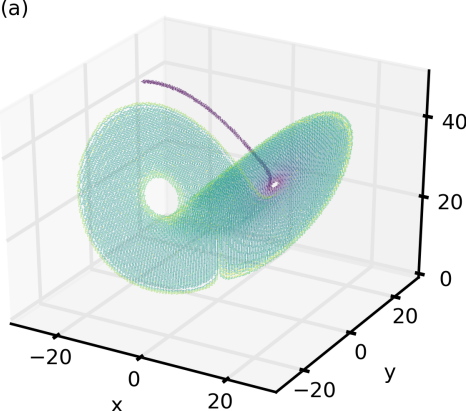

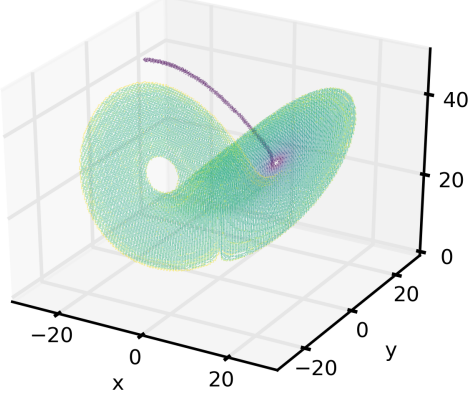

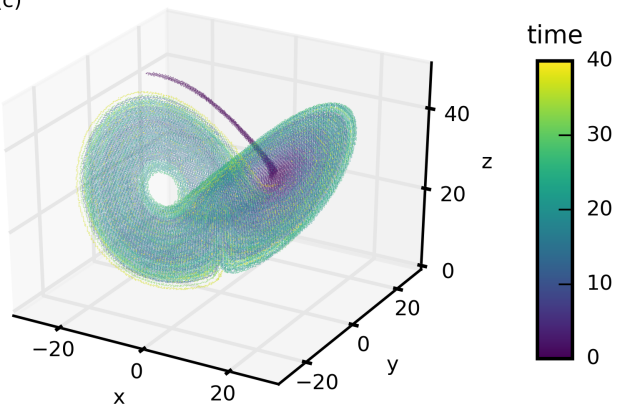

Figure 6. Attractor's points visited by 100-particles ensembles initialized with the same random initial condition (strong noise case $\Upsilon=100$ ) for $t \in[0,40]$ for the deterministic Lorenz system (a), the diffusive "LES-LZ" Lorenz system (b) and the "LUS-LZ" stochastic Lorenz system under location uncertainty (c). The color encodes the time necessary to reach a given point of the attractor for the first time.


ive 7. Mean attractor visiting rate (see text) up to $t=40$ computed for 10,000 realizations ( 100 ensembles of 100 particles) for the deterministic Lorenz system (black); diffusive "LES-LZ" Lorenz system (green) and the "LUS-LZ" stochastic Lorenz under location uncertainty " (red); the \pm standard deviations are superimposed in ghter color: (a) strong noise case; (b) small noise case.

In the strong noise case, the visiting rate significantly differs for the three systems. As previously observed, the LES-LZ shows some

ficulties to efficiently explore the attractor. A significant part of the trajectories remains close to the equilibrium points. On average, s than $5 \%$ of the attractor has been visited at time $t=40$. The LZ system certainly performs better, but several configurations remains in the equilibrium basin. On average, about $7 \%$ of the attractor have been explored in the same lapse of time. The stochastic system provides much better results. It enables to explore a much greater part of the attractor (nearly $25 \%$ in average) for the same number of realizations. In the small noise case, as could have been anticipated, the LES-LZ results are much closer to the LZ one. It can be noted thit almost the same portion of the attractor, as in the strongly perturbed case, have been explored by both LES-LZ and LZ. Therefore a stronger perturbation of the initial condition of the classical Lorenz system only results in a small increase of the attractor visit. The ndard deviation associated to the deterministic systems (LES-LZ and LZ) is not significantly strengthened by a strong perturbation of the initial condition. The LUS-LZ, even in a small noise configuration, shows a remarkable ability to visit a larger portion of the iractor $(>10 \%)$.

It can be noticed that for LUS-LZ and LZ the variance of the visiting rate grows rapidly at short time while it strongly decreases at the end of the temporal window. The large variance increase at the beginning is connected to the different initial conditions (randomly drawn on the attractor). Some ensembles will reach the regions of bifurcation of the attractor more rapidly than others, depending on where they have been started. When visiting these regions, particles of a given ensemble are sent to very different trajectories. Therefore, for such ensembles, the visiting rate increases rapidly at short time. Other ensembles will take longer to reach the bifurcations, their particles stay close together for a longer time and the visiting rate increases slowly. But, given enough time and for both models, most ensembles go through the bifurcations, spread and explore a similar amount of the attractor the initial condition has been forgotten 
and the variance of the visiting rate decreases. The stochastic model is advantaged by the noise and continues to explore faster than the deterministic one even in a low noise context (the mean visit rate for LUS-LZ increases faster than that of $\mathrm{LZ}$ at $\mathrm{T}=40$ ). At the end of temporal window, the variance of LUS-LZ remains also higher than the variance of LZ, especially for strong noise. For high diffusion the LES-LZ keeps a high variance as some trajectories remain stuck in the attraction bassin of the equilibrium points.

\section{Conclusion}

As considered for this reduced system, the proposed stochastic strategy demonstrates great potential to model geophysical flows. The resulting stochastic system helps to very efficiently explore the entire dynamical landscape of the flows. Without considering a large computational load, a traditional diffusive setting appears more hazardous to use. This is especially true when a significant diffusion is studied. In that case, a purely diffusive subgrid model shows limited performances, and implies supplementary computational efforts. I. rapidly reaches the burdens of almost fully resolved systems ! As rigorously derived, the stochastic strategy helps to avoid eventual pitfalls leading to strongly biased scenarios from insufficient exploration of the phase space dynamics. As also tested, the addition of an enpirical stochastic forcing barely constitutes an acceptable solution, as possibly leading to a bad representation of the target system. P. F om these results, it thus appears mandatory to more systematically promote the derivation of proper stochastic representations of the classical geophysical systems for climatic analysis of geophysical flows, following geometric mechanics and variational principle Holm (2015), or the location uncertainty formalism as developed here above.

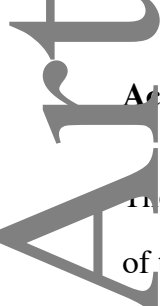

A/knowledgements

e authors are very grateful to Dan Crisan and Darryl Holm for valuable discussions on this work. We also acknowledge the support of the ESA DUE GlobCurrent project, the "Laboratoires d'Excellence" CominLabs, Lebesgue and Mer through the SEACS project.

\section{References}

B ge P, Pomeau Y, Vidal C. 1987. Order within chaos: Towards a deterministic approach to turbulence. John Wiley \& Sons: New York.

Berner J, Achatz U, Batte L, Camara ADL, Crommelin D, Christensen H, Colangeli M, Dolaptchiev S, Franzke C, Friederichs P, Imkeller P, Jarvinen H, Juricke , Kitsios V, Lott F, Lucarini V, Mahajan S, Palmer TN, Penland C, Storch JSV, Sakradzija M, Weniger M, Weisheimer A, Williams PD, Yano JI. 2017. Stochastic parameterization: Towards a new view of weather and climate models. Bull. Amer. Meteor. Soc. 98(3): 565-588.

Boussinesq J. 1877. Essai sur la th?orie des eaux courantes. Mémoires présentés par divers savants à l'Académie des Sciences, 23 (1): 1-680.

Chekroun M, Simonnet E, Ghil M. 2011. Stochastic climate dynamics: Random attractors and time-dependent stochastic climate dynamics: Random attractors allu ime-dependent invariant measures. Physica D 240: 1685-1700.

C ter C, Gottwald G, Holm D. 2017. Stochastic partial differential equations as a diffusive limit of deterministic Lagrangian multi-time dynamics. Technical report, arXiv:1706.00287.

C san D, Flandoli F, Holm D. 2017. Solution properties of a 3d stochastic Euler fluid equation. Technical report, arXiv:1704.06989.

Dellnitz M, Froyland G, Junge O. 2001. The algorithms behind gaio-set oriented numerical methods for dynamical systems. In: Ergodic theory, analysis, and efficient simulation of dynamical systems, Springer, pp. 145-174.

Dorfle M, Graham R. 1983. Probability density of the Lorenz model. Phys. Rev. A 27: 1096-1105.

nzke C, O'Kane T, Berner J, Williams P, Lucarini V. 2015. Stochastic climate theory and modeling. Wiley Interdisciplinary Reviews: Climate Change 6(1): $63-78$.

Guprts B, Holm D, Luesink E. 2017. Stochastic transport v fluctuation-dissipation noise in lorenz 63. Technical report, arXiv:1706.05882.

Holm D. 2015. Variational principles for stochastic fluid dynamics. Proc. R. Soc. A 471(ISSN: 1364-5021).

Kraichnan R. 1968. Small-scale structure of a scalar field convected by turbulence. Physics of Fluids (1958-1988) 11(5): 945-953.

Kraichnan R. 1994. Anomalous scaling of a randomly advected passive scalar. Physical review letters 72(7): 1016. 
Lorenz E. 1963. Deterministic nonperiodic flow. J. of atmos. Sci. 73(12): 130-141.

Majda A, Kramer P. 1999. Simplified models for turbulent diffusion: Theory, numerical modelling, and physical phenomena. Physics report 314: $237-574$.

Mémin E. 2014. Fluid flow dynamics under location uncertainty. Geophysical \& Astrophysical Fluid Dynamics 108(2): 119-146, doi:10.1080/03091929.2013. 836190.

Oksendal B. 1998. Stochastic differential equations. Spinger-Verlag.

Reeks M. 1983. The transport of discrete particles in inhomogeneous turbulence. J. Aerosol Sci. 14(6): 729-739.

Resseguier V, Mémin E, Chapron B. 2017a. Geophysical flows under location uncertainty, part I: Random transport and general models. Geophysical \& Astrophysical Fluid Dynamics 111(3): 149-176.

Resseguier V, Mémin E, Chapron B. 2017b. Geophysical flows under location uncertainty, part II: Quasi-geostrophic models and efficient ensemble spreading Geophysical \& Astrophysical Fluid Dynamics 111(3): 177-208.

Resseguier V, Mémin E, Chapron B. 2017c. Geophysical flows under location uncertainty, part III: SQG and frontal dynamics under strong turbulence. Geophysical \& Astrophysical Fluid Dynamics 111(3): 209-227.

R sseguier V, Mémin E, Heitz D, Chapron B. 2017d. Stochastic modelling and diffusion modes for proper orthogonal decomposition models and small-scale flow analysis. J. of Fluid Mech. 828: 888-917.

agorinsky J. 1963. General circulation experiments with the primitive equation: I. the basic experiment. Monthly Weather Review 91: 99-165.

S ra P, Newman M, Penland C, Sardeshmukh P. 2005. Multiplicative noise and non-gaussianity: A paradigm for atmospheric regimes? J. of Atmos. Sc. 62: $1391-1409$.
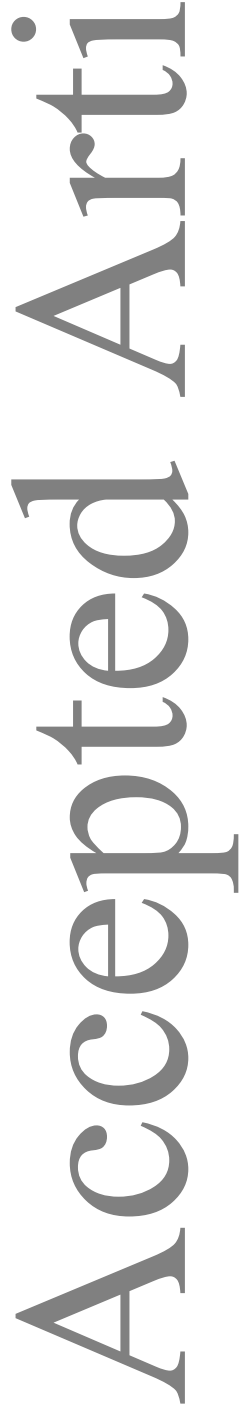